\title{
Turbulent amplification of magnetic fields in laboratory laser-produced shock waves
}

\author{
J. Meinecke ${ }^{1 \star}$, H. W. Doyle ${ }^{1}$, F. Miniati ${ }^{2}$, A. R. Bell ${ }^{1}$, R. Bingham ${ }^{3,4}$, R. Crowston ${ }^{5}$, R. P. Drake ${ }^{6}$, \\ M. Fatenejad ${ }^{17}$, M. Koenig ${ }^{8}$, Y. Kuramitsu ${ }^{9}$, C. C. Kuranz ${ }^{6}$, D. Q. Lamb7 , D. Lee ${ }^{7}$, M. J. MacDonald ${ }^{6}$, \\ C. D. Murphy ${ }^{10}$, H-S. Park ${ }^{11}$, A. Pelka ${ }^{8}$, A. Ravasio ${ }^{8}$, Y. Sakawa ${ }^{9}$, A. A. Schekochihin ${ }^{1}$, A. Scopatz ${ }^{7}$, \\ P. Tzeferacos ${ }^{1,7}$, W. C. Wan ${ }^{6}$, N. C. Woolsey ${ }^{5}$, R. Yurchak ${ }^{8}$, B. Reville ${ }^{1,12}$ and G. Gregori ${ }^{1,7 \star}$
}

X-ray ${ }^{1-3}$ and radio $^{4-6}$ observations of the supernova remnant Cassiopeia $A$ reveal the presence of magnetic fields about 100 times stronger than those in the surrounding interstellar medium. Field coincident with the outer shock probably arises through a nonlinear feedback process involving cosmic rays $\mathbf{s}^{2,7,8}$. The origin of the large magnetic field in the interior of the remnant is less clear but it is presumably stretched and amplified by turbulent motions. Turbulence may be generated by hydrodynamic instability at the contact discontinuity between the supernova ejecta and the circumstellar gas ${ }^{9}$. However, optical observations of Cassiopeia A indicate that the ejecta are interacting with a highly inhomogeneous, dense circumstellar cloud bank formed before the supernova explosion ${ }^{10-12}$. Here we investigate the possibility that turbulent amplification is induced when the outer shock overtakes dense clumps in the ambient medium ${ }^{13-15}$. We report laboratory experiments that indicate the magnetic field is amplified when the shock interacts with a plastic grid. We show that our experimental results can explain the observed synchrotron emission in the interior of the remnant. The experiment also provides a laboratory example of magnetic field amplification by turbulence in plasmas, a physical process thought to occur in many astrophysical phenomena.

High-resolution X-ray images and radio polarization maps of Cassiopeia A show two distinct strong magnetic field regions ${ }^{3-6,12}$. Narrow X-ray filaments, a fraction of a parsec in width, are observed at the outer shock rim at a radius of about $2.5 \mathrm{pc}$. These structures are produced by synchrotron radiation from ultrarelativistic electrons (with teraelectronvolt energy) and can be explained by magnetic fields of the order of $100 \mu \mathrm{G}$ or more $^{2,3}$. The interior of the remnant contains a disordered shell (about $0.5 \mathrm{pc}$ in width at a radius of $1.7 \mathrm{pc}$ ) of radio synchrotron emission by gigaelectronvolt electrons ${ }^{4}$. The inferred magnetic field in these radio knots is a few milligauss, about 100 times higher than expected from the standard shock compression of the interstellar medium ${ }^{15}$. Optical observations of Cassiopeia A show the presence of both rapidly moving $\left(5,000-9,000 \mathrm{~km} \mathrm{~s}^{-1}\right)$ and essentially stationary dense knots. Although the moving knots themselves have a high velocity, their overall pattern is nearly stationary ${ }^{10}$. This led to the suggestion ${ }^{10}$ that a dense pre-existing inhomogeneous stationary cloud bank could be present. New rapidly moving knots predominantly appear at a position broadly coincident with the shell of bright radio emission ${ }^{6}$. Sizes of the observed small-scale features within the shell range from 0.01 to $0.1 \mathrm{pc}$ arranged in larger patterns extending to $0.5-2 \mathrm{pc}$ (ref. 16). Interaction between the ejecta and the cloud bank may excite the turbulence that amplifies the magnetic field and makes Cassiopeia A an exceptionally bright radio source ${ }^{4}$. The interaction is akin to the Rayleigh-Taylor instability otherwise proposed as a source of turbulence and magnetic field at the contact discontinuity between the ejecta and the swept-up ambient medium ${ }^{9}$ except that the interaction begins with an already disturbed density configuration.

Our experiments focus on magnetic field amplification in a clumpy medium by making in situ magnetic field measurements in the turbulent wake of a laser-produced shock in a plasma. The interaction of a shock with large density perturbations is reproduced in the laboratory by passing a laser-produced shock in a plasma through a plastic mesh with $\ell=1.1 \mathrm{~mm}$ grid cell size, and 0.4 mm-thick wires (see Fig. 1 for details of the experiment). In situ magnetic field measurements are made in the turbulent wake of the shock. Using hydrodynamic scaling relations ${ }^{17,18}$, our experimental conditions at $0.3 \mu \mathrm{s}$ after the laser illumination can be scaled to Cassiopeia A signal to noise (SNR) with a probable age $t_{\mathrm{SNR}}$ of approximately 310 years, an expansion velocity $v_{\mathrm{SNR}}$ of about $4,700 \mathrm{~km} \mathrm{~s}^{-1}$ and a deceleration parameter $v_{\mathrm{SNR}} t_{\mathrm{SNR}} / R_{\mathrm{SNR}}$, where $R_{\mathrm{SNR}}$ is the shock radius, of about 0.6 (ref. 2; see Supplementary Information for the description of the scaling relations, and Supplementary Table 1). The same scaling relations imply the wire thickness in the mesh corresponds to $\sim 0.1 \mathrm{pc}$ in the SNR.

\footnotetext{
${ }^{1}$ Department of Physics, University of Oxford, Parks Road, Oxford OX1 3PU, UK, ${ }^{2}$ Department of Physics, ETH Zürich, Wolfgang-Pauli-Strasse 27, CH-8093 Zürich, Switzerland, ${ }^{3}$ Rutherford Appleton Laboratory, Chilton, Didcot OX11 OQX, UK, ${ }^{4}$ Department of Physics, University of Strathclyde, Glasgow G4 ONG, UK, ${ }^{5}$ Department of Physics, University of York, Heslington, York YO10 5D, UK, ${ }^{6}$ Atmospheric, Oceanic, Space Science, University of Michigan, 2455 Hayward Street, Ann Arbor, Michigan 48103, USA, ${ }^{7}$ Department of Astronomy and Astrophysics, University of Chicago, 5640 South Ellis Avenue, Chicago, Illinois 60637, USA, ${ }^{8}$ Laboratoire pour I'Utilisation de Lasers Intenses, UMR7605, CNRS CEA, Université Paris VI Ecole Polytechnique, 91128 Palaiseau Cedex, France, ${ }^{9}$ Institute of Laser Engineering, Osaka University, 2-6 Yamadaoka, Suita, Osaka 565-0871, Japan, ${ }^{10}$ School of Physics and Astronomy, University of Edinburgh, Edinburgh EH8 9YL, UK, ${ }^{11}$ Lawrence Livermore National Laboratory, Livermore, California 94550, USA, ${ }^{12}$ School of Mathematics and Physics, Queens University of Belfast, Belfast BT7 1NN, UK. *e-mail: jena.meinecke@physics.ox.ac.uk; g.gregori1@physics.ox.ac.uk
} 
a

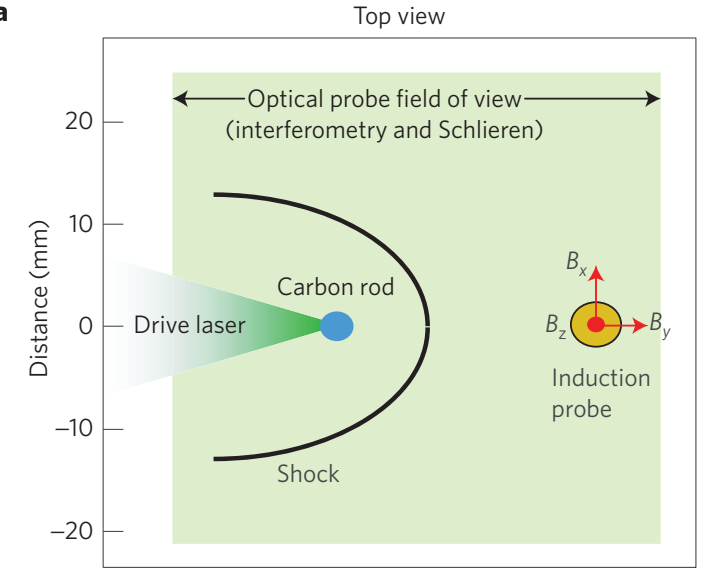

b

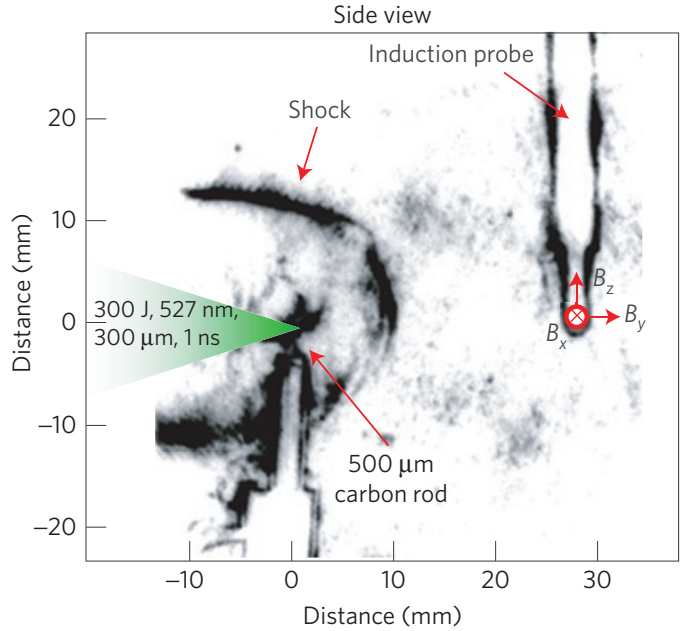

c

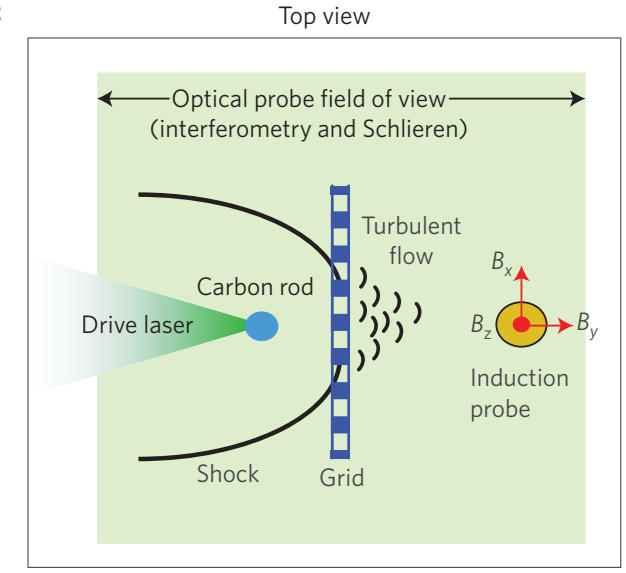

d

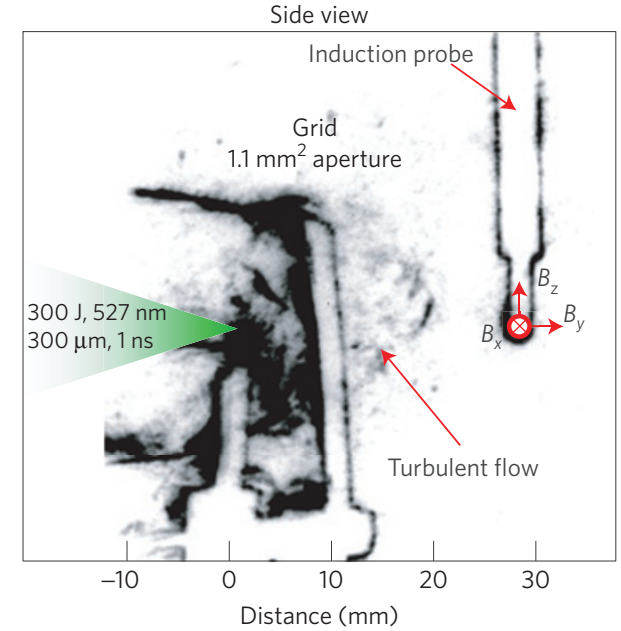

Figure 1 | Experimental configuration for the generation of shock-induced turbulence. The chamber is filled with argon gas at $P=1 \pm 0.2 \mathrm{mbar}$. Three frequency-doubled (527 nm wavelength) laser beams of the Vulcan laser facility are focused onto a 500- $\mu$ m-diameter carbon rod with a laser spot diameter of $300 \mu \mathrm{m}$. The total laser illumination is $300 \pm 30 \mathrm{~J}$ in a $1 \mathrm{~ns}$ pulse. The corresponding laser intensity is $/ \mathrm{L}=4 \times 10^{14} \mathrm{~W} \mathrm{~cm}{ }^{-2}$. The shock wave evolution was monitored using transverse interferometry and Schlieren with an optical probe (with $532 \mathrm{~nm}$ wavelength and $5 \mathrm{~ns}$ gate width). The Schlieren imaging technique measures density gradients. Thus, turbulent flow, with less distinct gradients, exhibits a reduced contrast. The interferometer was of Mach-Zehnder type with $\sim 50 \mathrm{~mm}$ field of view, and was used to provide the electron density. The induction coils are based on a modified design ${ }^{22,29}$ to achieve $\sim 100 \mathrm{MHz}$ bandwidth. They are placed at $3 \mathrm{~cm}$ (and at $4 \mathrm{~cm}$ for some laser shots) from the carbon rod position (that is, the centre of the initial blast). Each coil consists of 4 twisted pair coils wound around the axis of a $\sim 1 \times 1 \mathrm{~mm}^{2}$ plastic core. The voltage from the twisted pair loops is then differentially amplified to cancel any voltage induced from the plasma's electric field components, and the magnetic field is calculated using Faraday's law. The coils are protected from the surrounding plasma by a boron nitride tube. $\mathbf{a}$, Diagram of the experiment without the grid. $\mathbf{b}$, The same as in $\mathbf{a}$, but in the presence of a plastic grid placed at $1 \mathrm{~cm}$ from the carbon rod position. $\mathbf{c}$, Schlieren image at $t=300 \mathrm{~ns}$ after the laser shot and without the grid. $\mathbf{d}$, The same as in $\mathbf{c}$, but with the grid.

Wind tunnel experiments ${ }^{19}$ with a steady flow have shown that homogeneous turbulence is already well developed at distances $d \sim 20 \ell$, motivating us to take our field measurements predominantly at a distance of $2 \mathrm{~cm}$ downstream of the grid. Without the presence of the grid, the position $\left(R_{\mathrm{s}}\right)$ and properties of the shock wave have been monitored at various times using Schlieren, interferometry and spectroscopy diagnostics (Fig. 2). The shock closely follows a Sedov-Taylor solution ${ }^{20}$ with $R_{\mathrm{s}} \propto t^{2 / 5}$. Numerical simulations performed in one-dimensional (1D) spherical geometry with the collisional-radiative hydrodynamic code HELIOS, and in 2D-cylindrical geometry with the magnetohydrodynamic code FLASH (see Supplementary Information for details on the simulations) reproduce well the shock position, peak electron density and temperature values.

At the position of the grid $\left(R_{\mathrm{s} 0}=1 \mathrm{~cm}\right)$, the flow velocity of the shock is measured to be $v_{0} \sim 2 \times 10^{6} \mathrm{~cm} \mathrm{~s}^{-1}$ (Mach number $\sim 9$ ), although this increases on interaction with the grid. The Reynolds number corresponding to this shock radius is $\operatorname{Re}=v_{0} R_{\mathrm{s} 0} / \nu \sim 5 \times 10^{4}$
( $v \approx 40 \mathrm{~cm}^{2} \mathrm{~s}^{-1}$ is the plasma viscosity), and the magnetic Reynolds number is $\mathrm{Rm}=v_{0} R_{\mathrm{s} 0} / \eta \sim 3-6$, with magnetic diffusivity $\eta \approx 4 \times 10^{5} \mathrm{~cm}^{2} \mathrm{~s}^{-1}$ (more details are given in the Supplementary Information). As shown in earlier hydrodynamic simulation ${ }^{21}$ and in FLASH magnetohydrodynamic simulations of the experiment, for large Re, the grid acts as a channel, which accelerates the flow as it passes through the pores, after which the flow velocity decreases. This can be seen in Fig. 1, where, with the grid, the turbulent flow seems to advance more rapidly towards the position of the magnetic field diagnostics. From Schlieren imaging, for all experiments with the grid, the shock is observed to arrive at the position of the probe at $t \gtrsim 1.5 \mu$ s.

Figure 3 shows that in the case of a grid, the perpendicular component $\left(B_{\perp}^{2}=B_{x}^{2}+B_{z}^{2}\right.$ in Fig. 1) of the magnetic field is 2-3 times larger than without it. Previous results ${ }^{22}$ have indicated that perpendicular magnetic fields are produced at the shock front through the Biermann battery mechanism ${ }^{23}$ owing to misaligned pressure and density gradients. We find that for shocks passing 

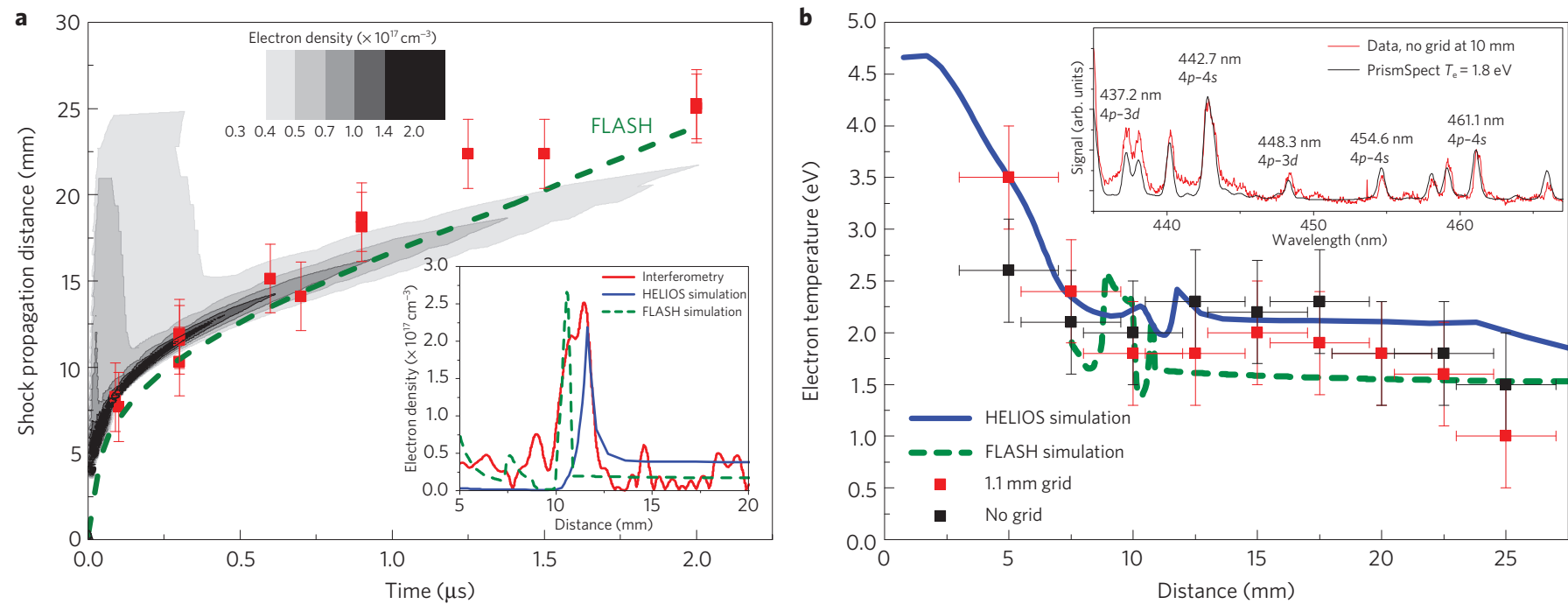

Figure $\mathbf{2}$ | Characterization of the shocked plasma. a, Measurement of the shock wave position versus time in the absence of a grid obtained from Schlieren data (red squares), 1D HELIOS simulations (contours) and 2D FLASH simulations (dashed green line). The inset shows the measured density profile obtained through interferometry at $t=300 \mathrm{~ns}$ compared with simulation predictions. The estimated maximum error of $\pm 2 \mathrm{~mm}$ in the experimental position is due to the uncertainty in the optical images to define the exact location of the shock front. $\mathbf{b}$, With reference to Fig. 1, the horizontal mid-plane joining the carbon rod position and the tip of the magnetic induction coils has been imaged onto a $50 \mu \mathrm{m}$ slit of a visible spectrometer coupled to a gated CCD (charge-coupled device) camera. This provides space- and time-dependent temperature information. The gating time of the CCD camera was 20 ns. The panel shows the measured electron temperature $\left(T_{\mathrm{e}}\right.$ ) profiles at $t=300$ ns without (black symbols) and with (red symbols) a grid obstacle in the flow. The experimental temperature values are compared with HELIOS and FLASH simulations. The low value of $T_{\mathrm{e}}$ near the target in the FLASH simulation is an artefact due to expansion of the cold carbon material; we therefore omit this part of the $T_{\mathrm{e}}$ profile in the graph. The inset shows the measured spectral lines at $1 \mathrm{~cm}$ from the carbon rod position (averaged over $0.2 \mathrm{~cm}$ ). The ratio of the emission lines has been fitted with the collisional-radiative code PrismSPECT (ref. 30). In the density region shown in $\mathbf{a}$, the line ratios are weakly dependent on the electron density, thus providing a good measurement of $T_{\mathrm{e}}$ alone. The error in the reported temperature values has been estimated from the small variations in the calculated spectral line shapes with changes in $T_{\mathrm{e}}$ of $\pm 0.5 \mathrm{eV}$.

through a grid, magnetic field amplification continues to occur long after the shock has passed, suggesting that an alternative mechanism is at play. This is attributed to the fact that the downstream flow is highly turbulent as a result of the shock-grid interaction. To confirm that the observed field amplification does not depend on the Biermann battery generation at the laser spot, we have run FLASH simulations with the latter process turned on only during the first $30 \mathrm{~ns}$ and switched off afterwards (Supplementary Fig. 1). This shows that the measured magnetic field is indeed enhanced as the shock passes through the grid owing, mainly, to the induced turbulence. Assuming a downstream flow velocity of the order $10^{5}-10^{6} \mathrm{~cm} \mathrm{~s}^{-1}$, the field is gradually amplified over a distance of $\lesssim 1 \mathrm{~cm}$ from the grid, before arriving at the probe, further supporting the mechanism of turbulent field amplification occurring by differential rotation induced by the mesh (Supplementary Movies 1 and 2).

The vorticity induced as the shock overtakes the grid acts as a seed for turbulent generation of magnetic field on a smaller scale. Beyond the grid, the vorticity is stretched in the direction of the flow, so that the spatial scale of the largest eddies is $L_{\mathrm{e}} \sim 7 \mathrm{~mm}$ by the time the shock reaches the induction probe (Fig. 3). As the magnetic Prandtl number is $\mathrm{Pm}=\mathrm{Rm} / \mathrm{Re}=v / \eta \approx 10^{-4}$, substantially lower than in the SNR case, the resistive scale lies well above the viscous scale and in fact above the energy-containing scale at which the inertial range starts (see below). Resistive diffusion of the magnetic field is increasingly important on smaller scales, although our measured increase in the magnetic field shows that it is not dominant at the scales of interest. Indeed, the measured $k$-spectrum of the magnetic field is consistent with the Golitsyn $\left(k^{-11 / 3}\right)$ powerlaw dependence ${ }^{24,25}$, characteristic of magnetic fluctuations at low Pm (refs 25-27; Fig. 4). This suggests that turbulent motions are present and amplifying the field at subresistive scales. With a small magnetic Reynolds number, the amplification is due to the stochastic tangling of an imposed field, $B_{0}$, by turbulent motions, and the saturated level is set by balancing this effect with Ohmic diffusion $^{25-27}$. In our experiment, $B_{0}$ is the Biermann-batterygenerated baroclinic magnetic field in the homogeneous flow. The amplified field scales as $|\delta B| \sim \operatorname{Rm}\left|B_{0}\right|$, and it remains dynamically unimportant because the Alfvén speed is smaller than the flow velocity $\left(v_{\mathrm{A}} \sim 400-1,400 \mathrm{~cm} \mathrm{~s}^{-1}\right)$.

FLASH simulations without a grid show that the shock becomes unstable when it is overtaken by the compositional discontinuity between the vaporized carbon target material and the gas, generating vorticity, and therefore magnetic fields, at spatial scales $\lesssim 3 \mathrm{~mm}$ (Fig. 3). This is the origin of the $k$-spectrum of the magnetic field without a grid. Simulations with a grid present show that the size of the largest eddies is $\sim 2$ times larger, the flow velocity is $\sim 50 \%$ larger, and thus the magnetic Reynolds number is $\sim 3$ times larger. This is consistent with the higher magnetic field seen in the experimental data.

Figure 3 shows that amplification occurs over distances much larger than the aperture of the grid. The slope of the normalized twopoint cross-correlation function of the measured magnetic field at $d=2 \mathrm{~cm}$ and $d=3 \mathrm{~cm}$ from the grid position (see Supplementary Information for the mathematical definition and Supplementary Fig. 2) shows an amplification rate $\gamma=4 \times 10^{5} \mathrm{~s}^{-1}$. This rate at the outer scale must be comparable to the Ohmic dissipation rate. Estimating this scale to be $\sim L_{\mathrm{e}}=7 \mathrm{~mm}$ (Fig. 3), we measure $\eta / L_{\mathrm{e}}^{2} \approx \gamma$. This timescale gives a good order-of-magnitude estimate of the effective stretching rate of the chaotic flow behind the grid and therefore of the typical minimal rate of magnetic field amplification one should expect in an astrophysical plasma, where resistivity is low and $\mathrm{Rm}$ is large. From our scaling relations, this laboratory stretching rate corresponds to a growth rate $\gamma_{\mathrm{SNR}} \sim$ $0.4 \times 10^{-3} \mathrm{yr}^{-1}$ on spatial scales $\sim 1 \mathrm{pc}$ in the SNR. At smaller scales in our experiment, where resistivity dominates, there are 
a

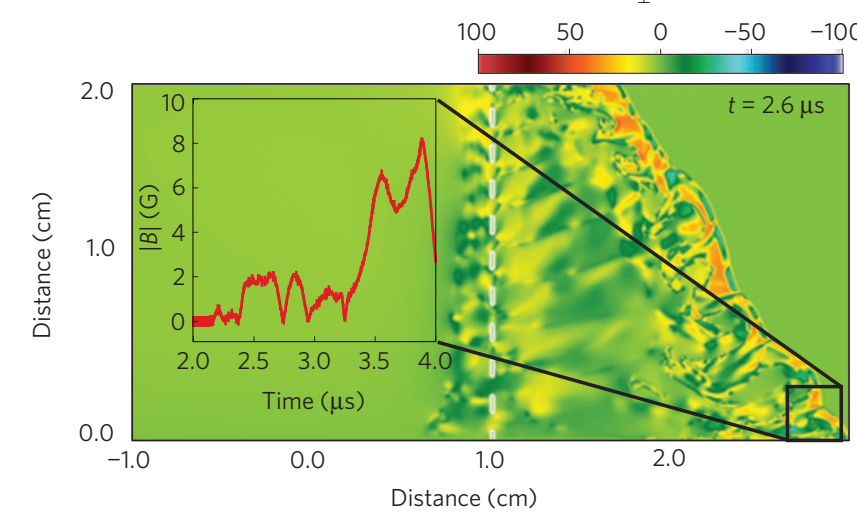

b

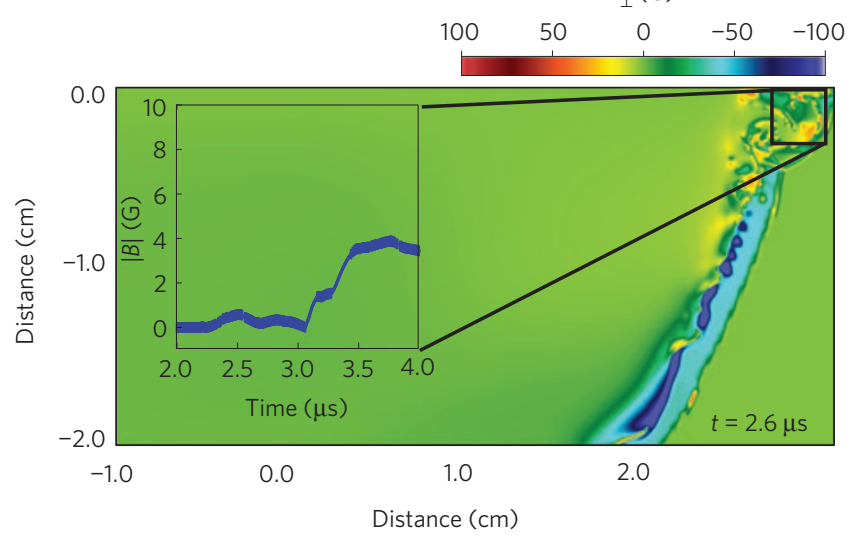

c

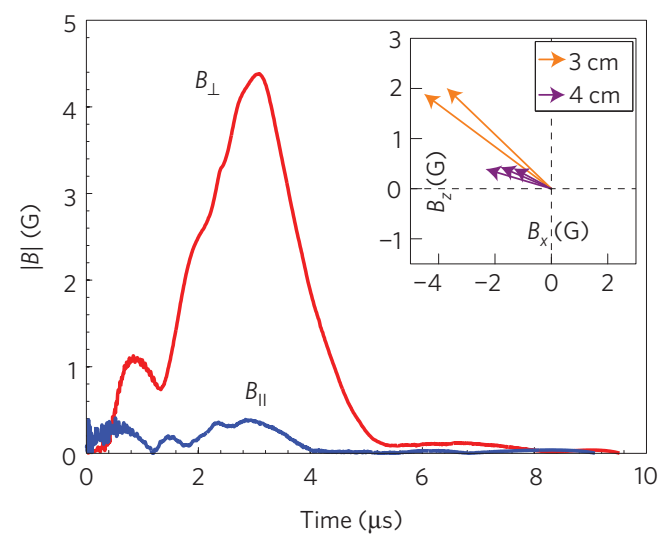

d

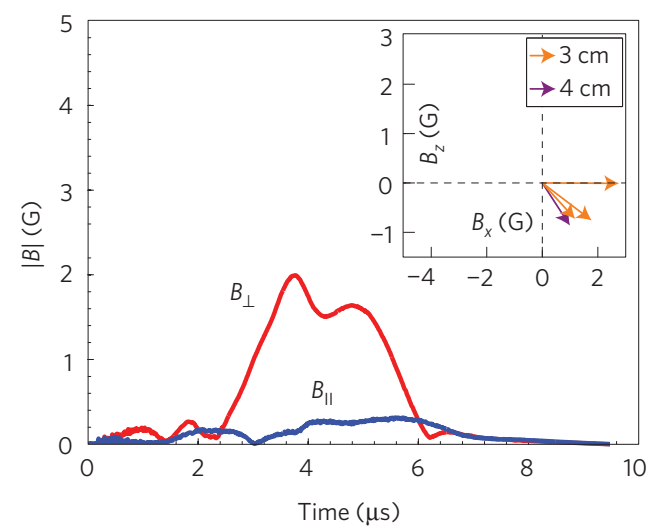

Figure 3 | Time evolution of the magnetic field. $\mathbf{a}$, FLASH simulation of the shock propagation through a grid at $t=2.6 \mu \mathrm{s}$. The inset shows the time variation of the magnetic field at $2.8 \mathrm{~cm}$ from the grid position along the symmetry axis (averaged over a $3 \mathrm{~mm} \times 3 \mathrm{~mm}$ volume). $\mathbf{b}$, The same as in $\mathbf{a}$, but with no grid. c, The magnetic field measured at $3 \mathrm{~cm}$ from the carbon rod with components along the axis as shown in Fig. 1 for the grid case. The time resolution is $10 \mathrm{~ns}$. The magnetic field has been extracted from the coil voltage by using a fast Fourier transform technique ${ }^{29}$. We estimate error in the magnetic field traces to be $\pm 0.3 \mathrm{G}$. Initial ( $t<100 \mathrm{~ns}$ ) high-frequency noise due to the laser-plasma interaction with the carbon rod has been filtered out in the extraction of the magnetic field. The inset shows the projection of the magnetic field onto the $x$ - $z$ plane at the time of its maximum intensity. Each vector corresponds to different shots. Data obtained at a distance of $4 \mathrm{~cm}$ from the carbon rod are also shown in the inset. In this case, the field magnitude is considerably reduced. $\mathbf{d}$, The same as in $\mathbf{c}$ but without the grid, as illustrated in Fig. 1. The measured signal starts earlier in the case with a grid than in the case without a grid for two reasons. First, the shock is accelerated as it passes through the grid, owing to the Bernoulli effect, whereas in the case without a grid, it is not. Second, the grid shadows the gas beyond it from the high-temperature radiation emitted at early times. Thus, in the case with a grid a significant fraction of the gas beyond the grid is not heated, and so has a higher diffusivity. This effect is visible in the measured temperature profiles of Fig. $2 \mathrm{~b}$.

still sustained magnetic fluctuations (Fig. 4). The timescale for the resistivity-chaotic-tangling balance to be established at those scales is $\eta / \ell_{\mathrm{e}}^{2}$, where $\ell_{\mathrm{e}}$ is of order $1 \mathrm{~mm}$ (which is the induction coil size, and therefore the measurement resolution limit). The fact that the Golitsyn spectrum extends at least to these scales is an indirect confirmation that turbulence is established ${ }^{25}$, with inertialrange motions tangling the magnetic field much faster than the motions at the energy-conserving scale $\left(L_{\mathrm{e}}\right)$. At scales of order $\ell_{\mathrm{e}}$, the corresponding timescale is $\lesssim 50 \mathrm{~ns}$ in the laboratory, translating to $\lesssim 50 \mathrm{yr}$ in the SNR over distances $\sim 0.25 \mathrm{pc}$.

The observed variability of visible moving knots in the inner regions of the remnant is of the order of $10 \mathrm{yr}$ (ref. 10). The radio emission from Cassiopeia $\mathrm{A}$ can be characterized as a bright disordered ring of emission coincident with the cloud bank ${ }^{10}$ surrounded by a polarized radio plateau of lower brightness with a predominantly radially stretched magnetic field extending to the outer shock seen in X-rays ${ }^{7}$. X-ray emission similarly consists of an inner ring of non-thermal emission broadly coincident with the cloud shell and an outer rim revealing the position of the outer $\operatorname{shock}^{28}$. The outer $\mathrm{X}$-ray rim is thin because highenergy synchrotron-emitting electrons cool rapidly immediately downstream of the shock. The reverse shock, or shocks, may also accelerate the electrons needed for synchrotron emission at the inner ring.

Magnetic field amplification in Cassiopeia A can be thus understood as being partly due to amplification by cosmic rays at the shock and partly by the interaction, at a smaller radius, between ejecta and a clumpy medium. The latter process can be directly inferred from the experiment and its simulation. Our results are consistent with these observations, namely that a rotational flow, driven by a shock passing through a stationary density perturbation, is necessary to both amplify and sustain strong magnetic fields in an expanding magnetized plasma over distances corresponding to many times the scale of the initial density perturbation. Indeed, Fig. 3 shows a disordered magnetic field, stretched from the grid position to the outer shock, matching the extent of the observed synchrotron emission. On the other hand, as also indicated in Fig. 3, in the absence of such perturbations (that is, without a grid), the magnetic field remains confined only to the outer rim. Our experimental work thus agrees with earlier numerical modelling of shock waves interacting with a clumpy medium ${ }^{13-15}$, and it shows that magnetic fields can, on average, be amplified by the shockinduced rotational flow to values $\sim 3$ higher than expected from the jump conditions alone. 


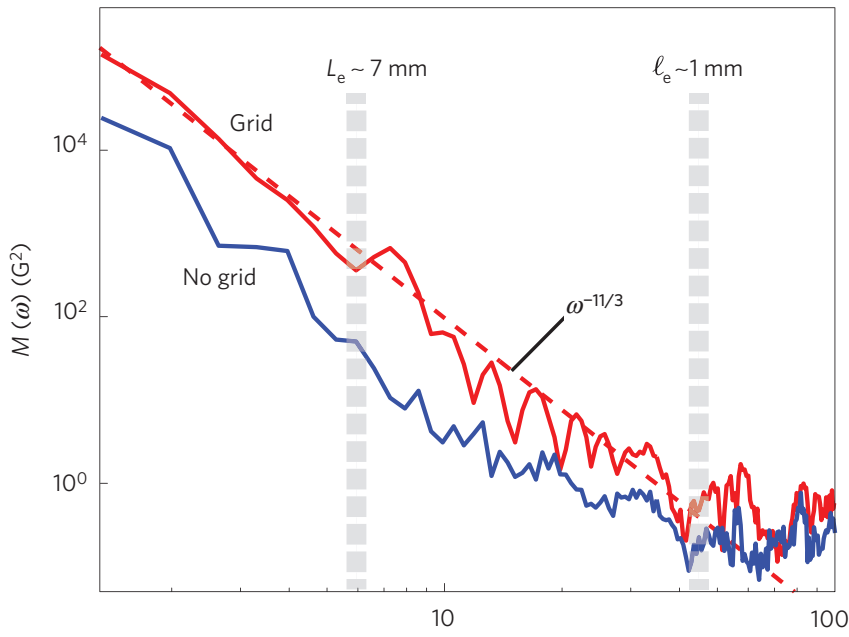

$\omega(\mathrm{MHz})$

Figure 4 | Frequency spectrum of the magnetic field. Plot of the measured magnetic energy spectrum $M(\omega)=|B(\omega)|^{2}$, where $B(\omega)$ is the fast Fourier transform of the total magnetic field for both the cases with (solid red line) and without (solid blue line) a grid. $M(\omega)$ is obtained by averaging all of the available laser shots with the induction coils placed at $3 \mathrm{~cm}$ from the carbon rod position. If we take at the induction coil position the flow velocity $v_{0} \sim 7 \times 10^{5} \mathrm{~cm} \mathrm{~s}^{-1}$, according to FLASH simulations with the presence of the grid, the corresponding spatial scales are indicated in the plot. $L_{\mathrm{e}}$ is the inferred energy-containing scale, that is, the scale of the largest eddies (Fig. 3), and $\ell_{\mathrm{e}}$ is the induction coil size, which determines the resolution limit. The measured spectrum shows a power law $\omega^{-11 / 3}$ (dashed red line). As the mean flow velocity is larger than the (subsonic) velocity fluctuations excited by the grid, then, according to Taylor's hypothesis, we obtain $\omega \sim v_{0} k$, where $k$ is the wavenumber of the magnetic fluctuations. As a result of the proportionality relation between frequency and wavenumber, the magnetic energy spectrum thus exhibits the expected Golitsyn $\left(k^{-11 / 3}\right)$ power-law dependence ${ }^{25-27}$

Received 22 October 2013; accepted 29 April 2014; published online 1 June 2014

\section{References}

1. Hwang, U., Holt, S. S. \& Petre, R. Mapping the x-ray-emitting ejecta in Cassiopeia A with Chandra. Astrophys. J. 537, 119-122 (2000).

2. Patnaude, D. J. \& Fesen, R. A. Proper motions and brightness variations of nonthermal x-ray filaments in the Cassiopeia A supernova remnant. Astrophys. J. 697, 535-543 (2009).

3. Stage, M. D., Allen, G. E., Houck, J. C. \& Davis, J. E. Cosmic-ray diffusion near the Bohm limit in the Cassiopeia A supernova remnant. Nature Phys. 2, 614-619 (2006)

4. Anderson, M. C. \& Rudnick, L. The deceleration powering of synchrotron emission from ejecta components in supernova remnant Cassiopeia A. Astrophys. J. 441, 307-333 (1995).

5. Anderson, M. C., Keohane, J. W. \& Rudnick, L. The polarization and depolarization of radio emission from supernova remnant Cassiopeia A. Astrophys. J. 441, 300-306 (1995).

6. Bell, A. R. Proper motions and temporal flux changes of compact features in Cassiopeia A at $5 \mathrm{GHz}$. Mon. Not. R. Astron. Soc. 179, 537-585 (1977).

7. Vink, J. \& Laming, J. M. On the magnetic fields and particle acceleration in Cassiopeia A. Astrophys. J. 584, 758-769 (2003).

8. Bell, A. R. Turbulent amplification of magnetic field and diffusive shock acceleration of cosmic rays. Mon. Not. R. Astron. Soc. 353, 550-558 (2004).

9. Gull, S. F. The X-ray, optical and radio properties of young supernova remnants. Mon. Not. R. Astron. Soc. 171, 263-278 (1975)

10. Van den Bergh, S. \& Dodd, W. W. Optical studies of Cassiopeia A. I. Proper motions in the optical remnant. Astrophys. J. 162, 485-493 (1970).

11. Chevalier, R. A. \& Oishi, J. Cassiopeia A and its clumpy presupernova wind. Astrophys. J. 593, L23-L26 (2003).
12. Borkowski, K. J., Szymkowiak, A. E., Blondin, J. M. \& Sarazin, C. L. A circumstellar shell model for the Cassiopeia A supernova remnant. Astrophys. J. 466, 866-870 ( 1996).

13. Gregori, G., Miniati, F., Ryu, D. \& Jones, T. W. Enhanced cloud disruption by magnetic field interaction. Astrophys. J. 441, L113-L116 (1999).

14. Giacalone, J. \& Jokipii, J. R. Magnetic field amplification by shocks in turbulent fluids. Astrophys. J. 663, L41-L44 (2007).

15. Guo, F. et al. On the amplification of magnetic fields by a supernova blast shock wave in a turbulent medium. Astrophys. J. 747, 98 (2012).

16. Milisavljevic, D. \& Fesen, R. A. A detailed kinematic map of Cassiopeia A's optical main shell and outer high-velocity ejecta. Astrophys. J. 772, 134 (2013).

17. Ryutov, D. D. et al. Similarity criteria for the laboratory simulation of supernova hydrodynamics. Astrophys. J. 518, 821-832 (1999).

18. Remington, B. A., Arnett, D., Drake, R. P. \& Takabe, H. Modeling astrophysical phenomena in the laboratory with intense lasers. Science 284, 1488-1493 (1999).

19. Comte-Bellot, G. \& Corrsin, S. The use of a contraction to improve the isotropy of grid-generated turbulence. J. Fluid Mech. 25, 657-682 (1966).

20. Ostriker, J. \& McKee, C. Astrophysical blastwaves. Rev. Mod. Phys. 60, $1-68$ (1988).

21. Naiman, H. \& Knight, D. D. The effect of porosity on shock interaction with a rigid porous barrier. Shock Waves 16, 321-337 (2007).

22. Gregori, G. et al. Generation of scaled protogalactic seed magnetic fields in laser-produced shock waves. Nature 481, 480-483 (2012).

23. Biermann, L. Über den Ursprung der Magnetfelder auf Sternen und im interstellaren Raum. Z. Naturforsch. A 5, 65-71 (1950).

24. Golitsyn, G. S. Fluctuations of the magnetic field and current density in a turbulent flow of a weakly conducting fluid. Sov. Phys. Dokl. 5, 536-539 (1960).

25. Schekochihin, A. A. et al. Fluctuation dynamo and turbulent induction at low magnetic Prandtl numbers. New J. Phys. 9, 300 (2007).

26. Moffatt, H. K. The amplification of a weak applied magnetic field by turbulence in fluids of moderate conductivity. J. Fluid Mech. 11, 625-635 (1961).

27. Odier, P., Pinton, J-F. \& Fauve, S. Advection of a magnetic field by a turbulent swirling flow. Phys. Rev. E 58, 7397-7401 (1998).

28. Helder, E. A. \& Vink, J. Characterizing the nonthermal emission of Cassiopeia A. Astrophys. J. 686, 1094-1102 (2008).

29. Everson, E. T. et al. Design, construction, and calibration of a three-axis, high-frequency magnetic probe (B-dot probe) as a diagnostic for exploding plasmas. Rev. Sci. Instrum. 80, 113505 (2009).

30. MacFarlane, J., Golovkin, I., Wang, P., Woodruff, P. \& Pereyra, N. Spect3d-a multi-dimensional collisional-radiative code for generating diagnostic signatures based on hydrodynamics and PIC simulation output. High Energy Dens. Phys. 3, 181-190 (2007).

\section{Acknowledgements}

We thank the Vulcan technical team at the Central Laser Facility of the Rutherford Appleton Laboratory for their support during the experiments; in particular, R. Clarke, M. Notley and R. Heathcote. A.R.B. acknowledges valuable discussions with H. Li (Los Alamos National Laboratory). The research leading to these results has received financial support from the European Research Council under the European Community's Seventh Framework Programme (FP7/2007-2013)/ERC grant agreements no. 256973 and 247039, LASERLAB-EUROPE grant agreement No. 284464, the US Department of Energy under Contract No. B591485 to Lawrence Livermore National Laboratory, and Field Work Proposal No. 57789 to Argonne National Laboratory. Partial support from the Science and Technology Facilities Council and the Engineering and Physical Sciences Research Council of the United Kingdom (Grant No. EP/G007187/1) is also acknowledged. The work of R.P.D., C.C.K., M.J.M. and W.C.W. was supported by the USDOE under grant DE-NA0001840.

\section{Author contributions}

G.G., D.Q.L, B.R. and F.M. conceived this project, and it was designed by G.G., J.M., B.R., C.D.M., R.B., A.A.S., N.C.W. and R.P.D. The Vulcan experiment was carried out by J.M., H.W.D., M.J.M., R.C., C.C.K., C.D.M., A.P. and W.C.W. The paper was written by J.M., G.G., H.W.D., A.A.S., A.R.B., D.Q.L., P.T. and B.R. The data were analysed by J.M. and H.W.D. Numerical simulations were performed by P.T. Further experimental and theoretical support was provided by R.B., R.P.D., M.F., M.K., Y.K., D.L., H-S.P., A.R., Y.S., A.S., P.T., N.C.W. and R.Y.

\section{Additional information}

Supplementary information is available in the online version of the paper. Reprints and permissions information is available online at www.nature.com/reprints. Correspondence and requests for materials should be addressed to J.M. or G.G.

\section{Competing financial interests}

The authors declare no competing financial interests. 